pulmonary complications occurred: $13.5 \%(n=10)$ developed pleural effusion requiring chest drain; 9.5\% $(\mathrm{n}=7)$ pneumonia; $4.1 \%(n=3)$ pulmonary embolus and 1.4\% $(n=1)$ sub-splenic

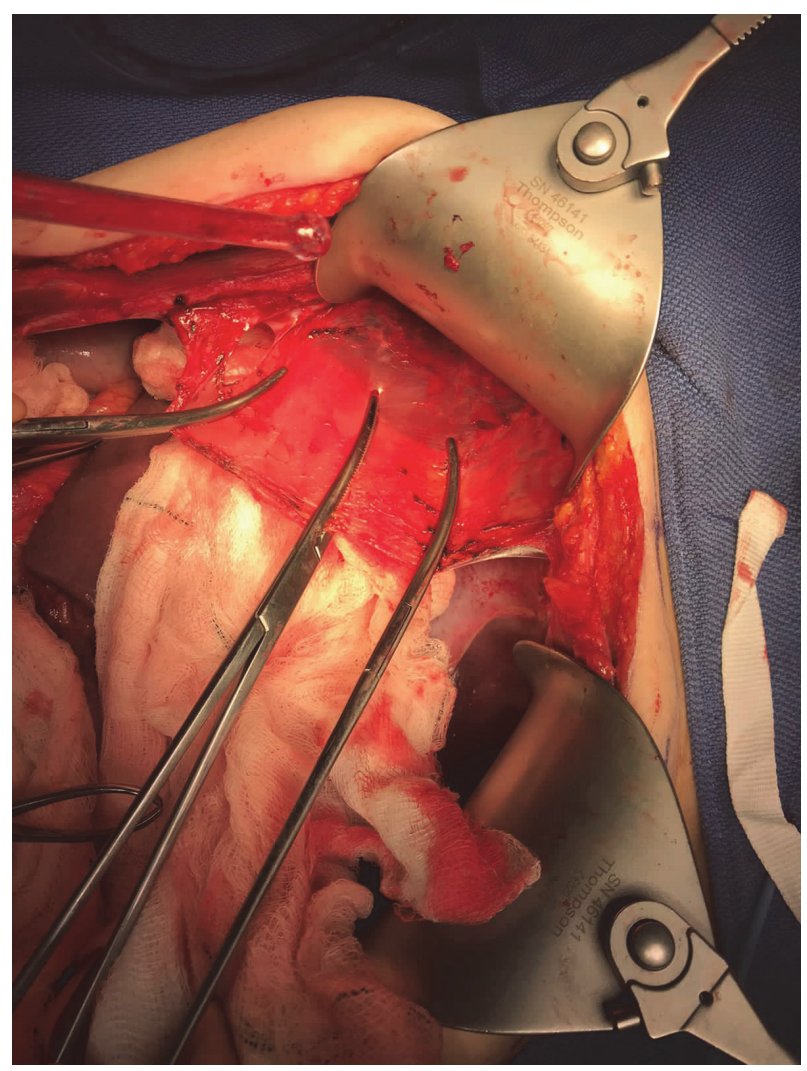

Abstract 530 Figure 1

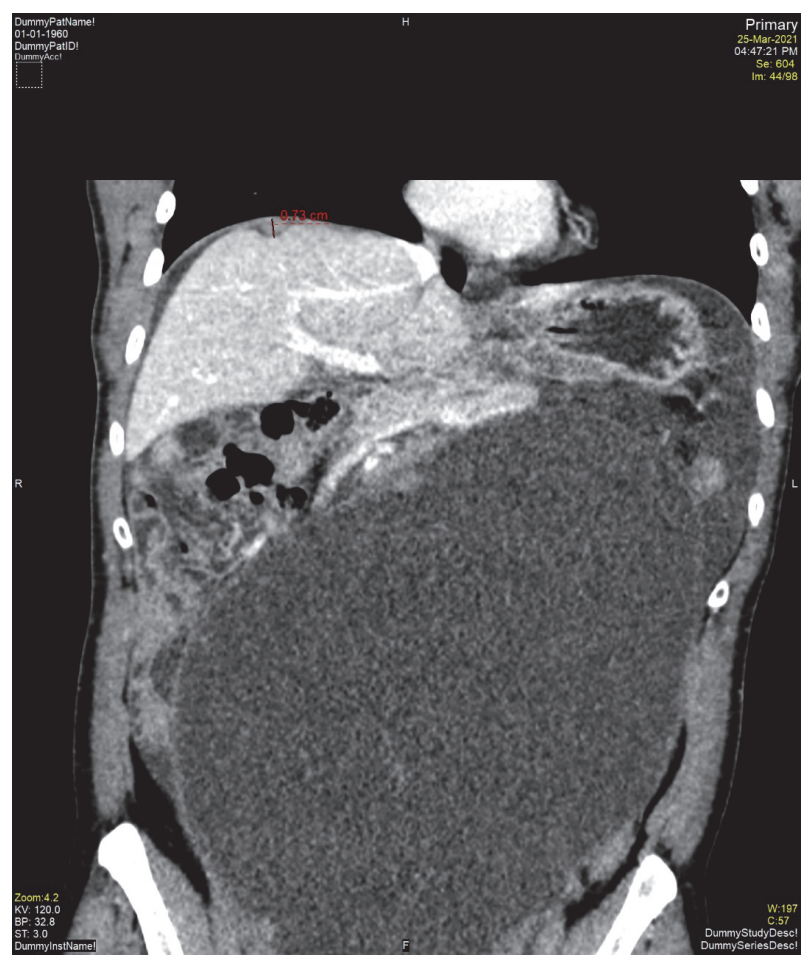

Abstract 530 Figure 2 haematoma. Median overall survival was 55 months $(95 \% \mathrm{CI}$ 33.4-77.6)

Conclusion* Surgeons should anticipate diaphragmatic disease in advanced ovarian or peritoneal cancer - and regard debulking of such an important prognostic factor. Diaphragmatic procedures appear feasible, without significantly increasing peri-operative morbidity in the context of ultra-radical surgery.

\section{BRCA STATUS AND PLATINUM-SENSITIVITY IN ADVANCED OVARIAN CANCER: A PATHOLOGICAL EVALUATION AFTER NEOADIUVANT CHEMOTHERAPY}

${ }^{1} \mathrm{R}$ Ergasti* ${ }^{*},{ }^{1} \mathrm{R}$ Tudisco, ${ }^{1} \mathrm{C}$ Marchetti, ${ }^{2} \mathrm{~A}$ lervolino, ${ }^{1,2} \mathrm{~A}$ Fagotti, ${ }^{1,2} \mathrm{G}$ Scambia. ${ }^{1}$ Fondazione Policlinico Universitario Agostino Gemelli, IRCCS, Department of Woman, Child, and Public Health, Rome, Italy; ${ }^{2}$ Catholic University of Sacred Heart, Rome, Italy

\subsection{6/ijgc-2021-ESG0.421}

Introduction/Background* To investigate the association between the presence of BRCA1/2 mutations and the chemotherapy response score (CRS) in patients with advanced stage high-grade serous ovarian cancer (HGSOC) undergoing neoadjuvant chemotherapy (NACT) and interval debulking surgery (IDS).

Methodology We retrospectively collected patients with unresectable HGSOC, who were assessed for BRCA1/2 mutational status and underwent 3 or 4 cycles of NACT, in our Institution from 2016 to 2020. All patients received germline test on peripheral blood sampling or somatic test at diagnostic biopsy. In all patients, the omental specimen obtained at IDS was evaluated according to Bohm's CRS System.

Result(s)* Of 184 patients analyzed, 69 were BRCA1/2 mutation carriers (37.5\%) and 115 were wild type (62.5\%). In the wild type patients group, 80 patients had a CRS 1/2 (69.6\%) and 35 patients had a CRS $3(30.4 \%)$. In the BRCA1/2 mutation carriers group, 38 patients had a CRS $1 / 2(55.1 \%)$ and 31 had a CRS $3(44.9 \%),(p=0.034)$.

Conclusion* BRCA1/2 mutational status is significantly associated with a different response rate to chemotherapy, as assessed according to Bohm's CRS System. Whereas in BRCA1/2 mutation carriers there does not appear to be a greater predisposition to achieve a CRS 3 than a CRS $1 / 2$, wild type patients appear to be more likely to have a CRS $1 / 2$.

\section{IDENTIFICATION OF MIRNA PANEL IN DIFFERENT TYPES OF ENDOMETRIOSIS AND OVARIAN CANCER}

${ }^{1} \mathrm{M}$ Szubert*, 'A Nowak, ${ }^{1} \mathrm{JR}$ Wilczynski, ${ }^{2} \mathrm{~B}$ Szymanska, ${ }^{3} \mathrm{D}$ Domanska-Senderowska. ${ }^{1}$ Medical University of Lodz, Poland, I Department of Gynecology and Obstetrics, Clinic of Surgical and Oncologic Gynecology, Lodz, Poland; ${ }^{2}$ The Central Scientific Laboratory (Corelab) Medical University of Lodz, Lodz, Poland; '3aklad Biomedycyny i Genetyki, Lodz, Poland

\subsection{6/ijgc-2021-ESGO.422}

Introduction/Background* Development of EAOC (Endometriosis associated ovarian cancer) has been intensively studied in the last few years. Epigenetic regulation of genes could play an important role in this process. The objective of this study was to identify miRNAs which expression was dysregulated both in EAOC and different types of endometriosis. 